\title{
Vehicle Detection Method based on Adap- tive Background Updating Algorithm
}

\author{
Guo Lin $^{1}$ ShenXiang-hui ${ }^{2}$ \\ ${ }^{1}$ Engineering Research Center of Digital Audio\&Video Ministry of Education, Commu- \\ nication University of China, Beijing 100024 \\ ${ }^{2}$ Engineering Research Center of Digital Audio\&Video Ministry of Education, Commu- \\ nication University of China, Beijing, 100024
}

\begin{abstract}
This paper mainly researches and simulates the adaptive background updating algorithm based on Gaussian Mixture Model (GMM) in video vehicle detection, and it researches on some methods to extract the foreground. Frame difference method uses the brightness difference of two consecutive frames to analysis the motion characteristics and the approximate location and shape. The improved mage compensate method combines binary video and edge video. And by judging district size, erosion and dilation, disturbing lanes and noise points are successfully removed.
\end{abstract}

Keywords: Adaptive Background Update; Gaussian Mixture Model; Video Vehicle Detection; Foreground Extraction; Frame Difference Method; Image Compensate Method

\section{Introduction}

In the method of vehicle identification and detection, the main background updating algorithm includes the temporal average model (TAM) ${ }^{[1]}$, the single Gaussian model (SGM) ${ }^{[2]}$ and the Gaussian mixture model (GMM) ${ }^{[3]}$. GMM is an important adaptive background updating algorithm. Although the computation is large, the algorithm can better extract the background under complex conditions. The common moving object detect methods are frame difference method and background difference method. The former compares the consecutive frames and gets the moving objects, but is not applied to the objects with similar grey or vein information. The latter compares the grey difference of current frame and background frame and set threshold to judge the moving objects. It is commonly used in the situation with stable camera. For the problem of the light mutation or jitter of the camera, an article ${ }^{[4]}$ proposed a method of increasing background update rate, so that the foreground will be updated as background quickly. For the problem of moving target which remains still for a long time becomes background easily, some articles ${ }^{[5][6]}$ proposed ways of adjusting learning rate and module number to enhance the model's adaptability of the background change. This article is mainly focused on adaptive Gaussian mixture model. The model can extract vehicles in the background with leaves fluttering. In addition, this article also studies several foreground extraction method, comparing the characteristics of each foreground extraction method.

\section{Adaptive background updating al- gorithm based on Gaussian mixture model}

Adaptive background update algorithm based on Gaussian mixture model uses multiple Gaussian model to describe the color distribution of a pixel. The probably of the current pixel $\mathrm{X}_{\mathrm{t}}$ and the Gaussian 
distribution of the color distribution of each point is

$$
\begin{aligned}
& P\left(X_{t}\right)=\sum_{i=1}^{K} \omega_{i, t} \eta\left(X_{t}, \mu_{i, t}, \sum_{i, t}\right) \\
& \eta\left(X_{t}, \mu_{i, t}, \sum_{i, t}\right)=\frac{1}{(2 \pi)^{n / 2}\left|\sum_{i, t}\right|^{1 / 2}} e^{-\frac{1}{2}\left(X_{t}-\mu_{t, t}\right)^{T} \sum_{i, t}^{-1}\left(X_{t}-\mu_{i, t}\right)}
\end{aligned}
$$

$\omega_{i, t}, \mu_{i, t}, \sum_{i, t}$ represent each single model's weight, mean and width size of unimodal distribution respectively. $\mathrm{K}$ represents the number of pixel values of distributed peak, usually values between 3 and 5. It depends on the distribution of pixel value. The larger the value is, the larger the system's calculate complexity is.

In order to make the model comply with the actual pixel distribution law, we need to update the model's parameters according to each new pixel value. The parameter correction detect if it match the model first. If it matches

$\left|X_{t}-\mu_{i, t}\right|<\lambda \delta_{i, t}, i=1,2, \ldots, K$, this

pixel matches the model. $\lambda$ is a constant set by experience, generally values between 2 to 3 . Otherwise, if not matches with this formula, it does not match. Regarding the matching pixel, we need to update the model's parameter according to formula (2).

$\left\{\begin{array}{l}\omega_{k, t}=(1-\alpha) \omega_{k, t-1}+\alpha \\ \sigma_{i+1}(x, y)^{2}=(1-\alpha) \sigma_{i-1}(x, y)^{2}+\alpha \sigma_{i}(x, y)^{2} \\ \mu_{i+1}(x, y)=(1-\alpha) \mu_{i}(x, y)+\alpha I_{i+1}(x, y)\end{array}\right.$

$\alpha$ represents the weight update rate. It represents the background updating speed, usually values between 0 and 1 . In order to reduce the background noise, it values small generally, such as 0.05 . When we need to correct faster, larger $\alpha$ is adopted. $I_{i+1}(x, y)$ represents the grayscale of the new pixel in $(x, y)$ point.
If pixel $\mathrm{X}_{\mathrm{t}}$ does not match one of the Gaussian distributions, we will consider the new pixel does not contribute to this single model distribution. In this condition, we don't need to change the parameter of the Gaussian model, and only change the weight. The method is $\omega_{k, t}=(1-\alpha) \omega_{k, t-1}$.

If pixel $\mathrm{X}_{\mathrm{t}}$ does not match any of the Gaussian distributions, we need to add a new single model to it, and remove one Gaussian distribution from the original model. The requirement of the new model's parameter initialization is a smaller weight value and a larger variance. Otherwise, the original weights of each model need to be normalized.

To extract the background pixel, we need to range the pixel according to the priority $\omega / \sigma$ of each Gaussian model, and take the pre-B Gaussian distribution from the $\mathrm{N}$ Gaussian distribution as the background model.

$$
\begin{aligned}
& B=\arg \min _{b}\left(\sum_{k=1}^{b} \omega_{k}>T\right) \\
& \left|X_{i, t}-\mu_{i, t}\right|<2.5 \sigma_{i, t}
\end{aligned}
$$

$\mathrm{T}$ is the threshold of the background selection. It determines the number of the Gaussian mixture model in the background. If $\mathrm{T}$ is small, the background model is always single mode. If $\mathrm{T}$ is large, it can describe multimodal which is caused by repeating change of the background, such as the leaves shaking or waterlines fluctuations. If there are two or more colors as background on the same pixel, the effect will be clearer. If $X_{i, t}$ matches one background Gaussian distribution of the total $\mathrm{K}$ Gaussian distribution, this pixel point is detected as background point, otherwise as foreground point. 


\section{Adaptive background updating al- gorithm simulation based on Gauss- ian mixture model}

We use video sequence named road_car.avi, total frame is 874 , each frame is $320 * 240$ pixels. The operating system is Windows XP. The memory of the computer is $1.94 \mathrm{~GB}$. The simulation platform is Matlab7.5.0. The way of getting initial background is statistical histogram method.

Figure 1(b) is background based on adaptive weight values to be updated. Figure 1 (c) is the vehicle's foreground by judging whether matching. Figure $1(\mathrm{~d})$ is the initial background by the way of statistical histogram method, in order to obtain an accurate reference. It is clear that the foreground can be extracted by Gaussian mixture model in the case of multimodal. The update of background is more stable and the vehicle's foreground can be extracted more accurately. The accuracy of the extraction is decided by the matching threshold.

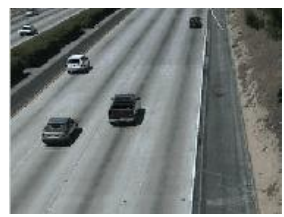

(a)Original video

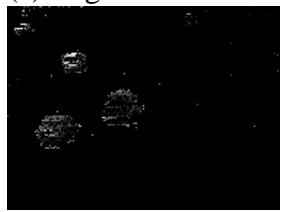

(b) Update background

(c) Extracted foreground (d)Initial background

Fig. 1: Simulation result based on GMM background updating algorithm

\section{Simulation of foreground extraction}

\subsection{Simulation of frame difference method}

We use the 120 video frames named viptraffic.avi to research the frame difference method. Firstly, we preprocess the original image, including color turning to grey, image smoothing. Processing result is indicated by figure 2(b). The adaptive background update is shown in figure 2(c), and it will change with real-time video. The foreground extracted by frame difference method is shown in figure 2(d). It is the difference between current video frame and the real-time update background. Frame difference method is applied to video with light changing background. The testing video background is stable relatively. Thus, we extract the foreground with frame difference method.

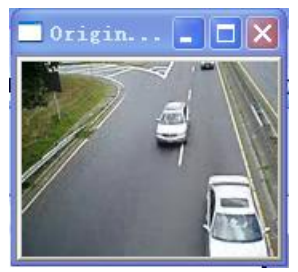

(a) Original video

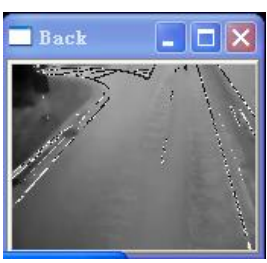

(c) Background

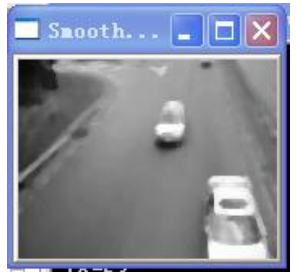

(b) Smoothed video

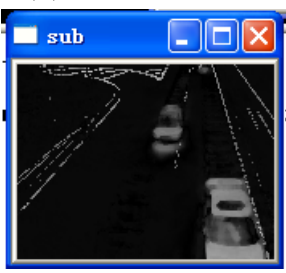

(d) Foreground
Fig. 2 Foreground simulation result based on frame difference method

\subsection{Simulation of image compensate method}

Image compensation is the adding result from edge video and binary video. Binary video is indicated by figure $3(\mathrm{~b})$. The background still extract some disturbing road lines, and the vehicles are not exactly extracted. Edge video is indicated by figure 3(c). There are still some disturbing lines after adding. However, through 
district size judgment, erosion and dilation, some little edge can be wiped off effectively and the exact vehicle image can be exactly extracted.

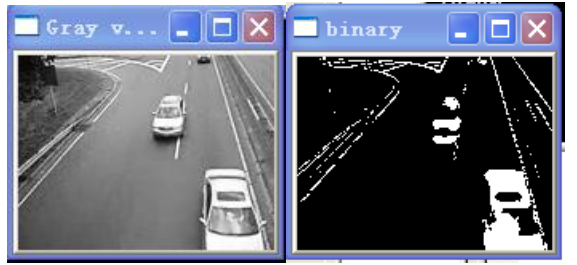

(a) Grey video

(b)Binary video

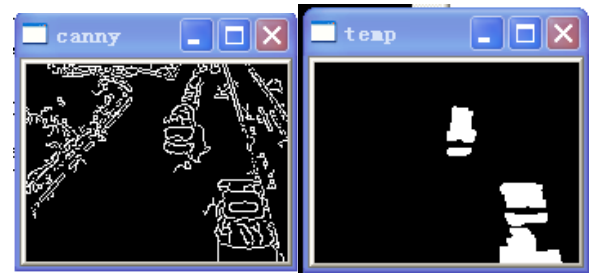

(c)Edge video

(d)Final result

Fig. 3 Image compensation video

\section{Conclusion}

In this paper, we research on the problem of vehicle video detection and realize the Gaussian mixture model algorithm. The Gaussian mixture method extracts the background with lots of noise points. The frame difference method can extract the vehicles but has some lanes disturbing. Comparing with it, the image compensate method can extract vehicles' basic shape and erase the road lanes. This paper research on videos with 120 frames, and the success rate is about 90 percent. This paper's algorithm is mainly used in the common road but not in the situation of stronger light or sheltering. The next research is mainly focused on improving the vehicle detection of light mutation, vehicle cover and the sudden movements of the stationary object.

\section{References}

[1] Friedman N, Russell S, “ Image segmentation in video sequences: a probabilistic approach," Proceedings of Thirteenth Conference on Uncertainty in Artificial Intelligence, Providence, Rhode Island, USA, Morgan Kaufmann Publishers. 175 181, 1997.

[2] Wren C R, Azarbayejani A, Darrell T, and Pentland A P.Pfinder, " Real-time tracking of the human body," IEEE Transactions on Pattern Analysis and Machine Intelligence. 19(7): 780 785, 1997.

[3] Stauffer C, Grimson W, “ Adaptive background mixture models for realtime tracking," Proceedings of IEEE Conference on Computer Vision and Pattern Recognition. Fort Collins, Colorado, USA, IEEE 246 252, 1999.

[4] Li Mingzhi, Ma Zhiqiang, and Shan Yong, " The adaptive background update of Gaussian mixture model under complex conditions," Computer Application, 2011.

[5] LEE D S, “ Effective Gaussian mixture learning," IEEE Transactions on Pattern Analysis and Machine Intelligence, 27 ( 5) : 827 - 832, 2005.

[6] ZORAN Z, " Recursive unsupervised learning of finite mixture models ," IEEE Transactions on Pattern Analysis and Machine Intelligence, 26( 5) : $651-656,2004$. 